\title{
Combined effects of 17 common genetic variants on type 2 diabetes risk in a Han Chinese population
}

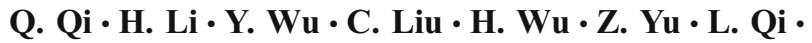 \\ F. B. Hu $\cdot$ R. J. F. Loos $\cdot$ X. Lin
}

Received: 24 February 2010 /Accepted: 24 May 2010 /Published online: 17 June 2010

(C) Springer-Verlag 2010

\begin{abstract}
Aims/hypothesis The recent advent of genome-wide association studies has considerably accelerated the identification of type 2 diabetes loci. We aimed to investigate the combined effects of multiple genetic variants, alone or in combination with conventional risk factors, on type 2 diabetes and diabetes-related traits in Han Chinese.

Methods We genotyped 17 variants in 17 loci in a population-based Han Chinese cohort including 3,210 unrelated individuals. A genetic risk score (GRS) was calculated on the basis of these variants. The discriminatory ability was assessed by the area under the receiver operating characteristics curve.
\end{abstract}

Electronic supplementary material The online version of this article (doi:10.1007/s00125-010-1826-5) contains supplementary material, which is available to authorised users.

Q. Qi $\cdot$ H. Li $(\bowtie) \cdot$ Y. Wu C C. Liu $\cdot$ H. Wu $\cdot$ Z. Yu $\cdot$ X. Lin $(\bowtie)$

Key Laboratory of Nutrition and Metabolism,

Institute for Nutritional Sciences,

Shanghai Institutes for Biological Sciences,

Chinese Academy of Sciences and Graduate School

of the Chinese Academy of Sciences,

294 Tai-Yuan Road,

Shanghai 200031, People's Republic of China

e-mail: lihx@sibs.ac.cn

e-mail: xlin@sibs.ac.cn

L. Qi F. B. Hu

Department of Nutrition, Harvard School of Public Health,

Boston, MA, USA

R. J. F. Loos

MRC Epidemiology Unit, Institute of Metabolic Science,

Addenbrooke's Hospital,

Cambridge, UK
Results The odds ratio for type 2 diabetes and hyperglycaemia with each GRS point (per risk allele) was 1.18 $\left(95 \%\right.$ CI $\left.1.12-1.23, p=1.3 \times 10^{-12}\right)$ and 1.12 (95\% CI 1.09 $\left.1.16, p=7.5 \times 10^{-14}\right)$, respectively. Compared with participants with GRS $\leq 11.0(7.63 \%)$, those with GRS $\geq 19.0$ (8.87\%) had a 4.58 -fold higher risk (95\% CI 2.49-8.42) of type 2 diabetes. The GRS also showed a significant association with lower beta cell function estimated by HOMA of beta cell function $\left(p=8.4 \times 10^{-10}\right)$. In addition, we observed significant interactive effects between GRS and $\mathrm{BMI}$ on fasting glucose and $\mathrm{HbA}_{1 \mathrm{c}}$ levels $(p=0.04$ and $p=0.03$ for interaction, respectively). Discrimination of diabetes risk was improved $(p<0.001)$ when the GRS was added to a model including clinical risk factors. The AUCs were 0.62 and 0.77 , respectively, for the GRS and conventional clinic risk factors alone, and 0.79 when the GRS was added.

Conclusions/interpretation In this Han Chinese population, the GRS of 17 combined variants modestly but significantly improved discrimination of the conventional risk factors for type 2 diabetes.

Keywords Chinese - Genetic risk score - Genetic variants . Population-based study - Receiver operating characteristic . Type 2 diabetes

$\begin{array}{ll}\text { Abbreviations } \\ \text { GRS } & \text { Genetic risk score } \\ \text { GWAS } & \text { Genome-wide association studies } \\ \text { HOMA-B } & \text { HOMA of beta cell function } \\ \text { HOMA-S } & \text { HOMA of insulin sensitivity } \\ \text { IFG } & \text { Impaired fasting glucose } \\ \text { NFG } & \text { Normal fasting glucose } \\ \text { SNP } & \text { Single nucleotide polymorphism }\end{array}$




\section{Introduction}

Genome-wide association studies (GWAS) have recently proven to be a powerful approach for identifying novel genetic risk variants that confer susceptibility to type 2 diabetes, with almost 20 loci discovered through GWAS in the last 3 years [1]. Most of these loci were identified in white Europeans; each of them only modestly increases disease risk and is of limited use in disease prediction. The combined information from these variants can improve identification of individuals who are at high vs those at low risk of type 2 diabetes [2-6]. However, these findings are not necessarily generalisable to other ethnic populations. In the present study, we examined the joint effects of 17 diabetes-associated variants on risk of type 2 diabetes and their discriminatory ability in presence of conventional risk factors such as BMI, family history and lifestyle in a population-based cohort of 3,210 unrelated Han Chinese.

\section{Methods}

Study population The study sample consisted of 3,210 individuals (1,423 men, 1,787 women) aged 50 to 70 years from the Study on Nutrition and Health of Aging Population in China. The study population, design, protocols and biochemical measurements of this study have been described previously $[7,8]$. BMI was calculated as weight $(\mathrm{kg}) /$ height $^{2}\left(\mathrm{~m}^{2}\right)$. HOMA of insulin sensitivity (HOMA-S) and beta cell function (HOMA-B) were estimated by Levy's computer model (http://www.dtu.ox.ac.uk/homacal culator/index.php, accessed 10 May 2010). Type 2 diabetes was defined as fasting glucose $\geq 7.0 \mathrm{mmol} / \mathrm{l}$ and/or a previous diagnosis of type 2 diabetes. Normal fasting glucose (NFG) and impaired fasting glucose (IFG) were defined as $<5.6 \mathrm{mmol} / 1$ and 5.6 to $<7.0 \mathrm{mmol} / \mathrm{l}$, respectively. The study was approved by the Institutional Review Board of the Institute for Nutritional Sciences, Shanghai, with written informed consent from all participants. The characteristics of the population are shown in Electronic supplementary material (ESM) Table 1.

Genotyping We successfully genotyped 17 previously reported type 2 diabetes-associated single nucleotide polymorphisms (SNPs; ESM Table 2) with a genotyping system (GenomeLab SNPstream; Beckman Coulter, Fullerton, CA, USA) or sequence detection system (ABI PRISM 7900 HT; Applied Biosystems, Foster City, CA, USA). The genotyping call rates were $>98 \%$ and the concordance rates were $>99 \%$ based on $12 \%$ duplicate samples $(n=384)$. The allele frequencies of all SNPs were comparable with the HapMap Han Chinese in Beijing, China database and were all in Hardy-Weinberg equilibrium $(p>0.05)$.
Genetic risk score computation The genetic risk score (GRS) was calculated by counting the number of risk alleles from the 17 variants, which produced a score ranging from 6 to 25. Participants for whom data on four or more genotypes were missing $(n=52)$ were excluded from the analyses. The GRSs of participants for whom data on one to three genotypes were missing $(n=384)$ were standardised to those of participants with complete data by the following equation: GRS $=$ (total number risk alleles $/$ number of non-missing genotypes $\times 2) \times 34$. In sensitivity analyses, we excluded all participants with missing genotypes, and observed similar results for the analyses that included all participants and those that excluded participants with missing data.

Statistical analysis Logistic regression was used to test the association of individual SNPs or GRSs with type 2 diabetes and IFG. Generalised linear regression was applied to quantitative trait analyses. Interactions between the GRS and conventional risk factors were tested by including the respective interaction terms in logistic and generalised linear regression models. The discriminatory values for type 2 diabetes risk were assessed by the area under the receiver operating characteristic curve in logistic regression models. All reported $p$ values are nominal and two-sided. Statistical analyses were performed with SAS version 9.1 (SAS Institute, Cary, NC, USA).

\section{Results}

The results of associations of individual SNPs with type 2 diabetes and its related traits, some of which have been published previously [8-10], are shown in ESM Table 2 and ESM Table 3. The SNPs in CDKAL1, PPARG, $K C N Q 1, H H E X / I D E, C D K N 2 A / B, T C F 2$ (also known as $H N F 1 B), K C N J 11, G C K R, I G F 2 B P 2$ and SLC30A8 were significantly associated with type 2 diabetes or hyperglycaemia $(p<0.05)$.

The distribution of the GRS was shifted to the right in type 2 diabetes patients compared with that in NFG individuals (ESM Fig.1a). Each additional point score increased risk of type 2 diabetes by 1.18 -fold $(p=1.3 \times$ $10^{-12}$ ) and risk of hyperglycaemia by 1.12 -fold $(p=7.5 \times$ $10^{-14}$; ESM Table 4). The risk of type 2 diabetes and hyperglycaemia increased across GRS quintiles (ESM Table 4). Compared with participants with a GRS $\leq 11.0$ (7.63\%), those with GRS $\geq 19.0(8.87 \%)$ had a 4.58 -fold (95\% CI 2.49-8.42) increased risk of type 2 diabetes and 2.8-fold (95\% CI 1.90-4.11) increased risk of hyperglycaemia (ESM Fig. 1b, c). Consistent with the above, individuals with higher GRS had significantly higher fasting glucose, $\mathrm{HbA}_{1 \mathrm{c}}$ and HOMA-S, and lower insulin, 

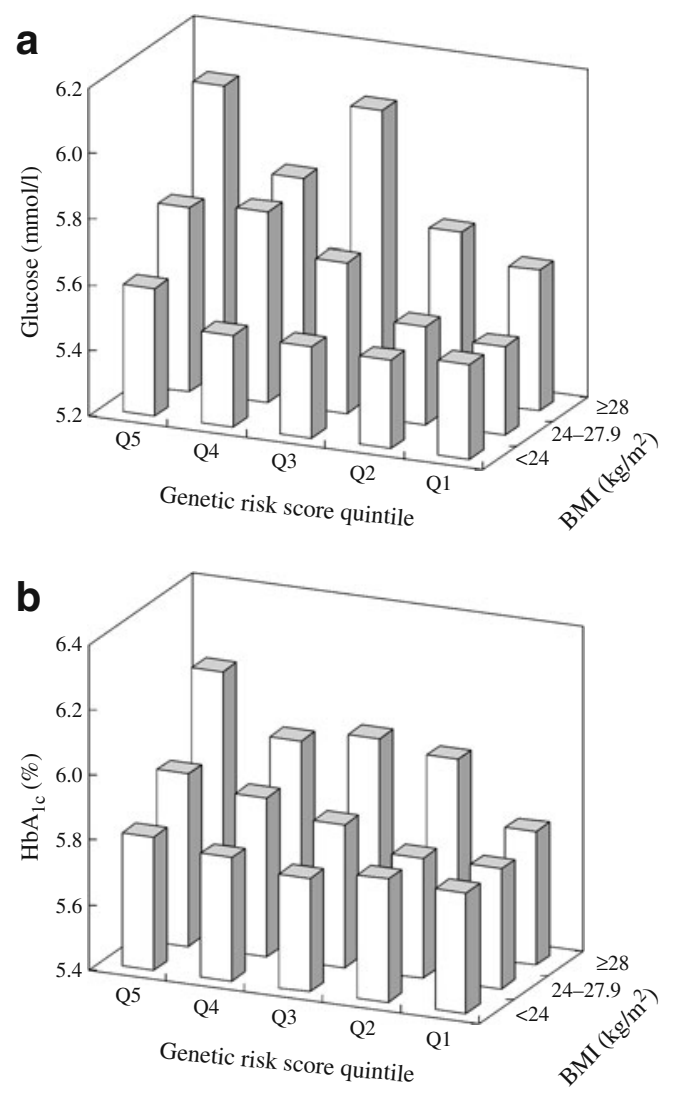

Fig. 1 Joint effects of BMI and GRS on (a) fasting glucose and (b) $\mathrm{HbA}_{1 \mathrm{c}}$ levels. Participants previously diagnosed with type 2 diabetes or receiving glucose-lowering treatment $(n=267)$ were excluded from the analyses. Data are means of fasting glucose and $\mathrm{HbA}_{1 \mathrm{c}}$ after adjustment for age, sex and region; (a) $p=0.04$ and (b) $p=0.03$ for interaction between BMI (three categories) and GRS

HOMA-B and BMI (ESM Table 5). Further adjustment for smoking, alcohol, physical activity and family history of diabetes did not substantially change the associations. Similar results were observed when analyses were performed using GRSs calculated by including the ten significantly associated SNPs only.

The risk of type 2 diabetes significantly increased with the presence of family history, higher BMI (three categories) and increasing quintiles of GRS (ESM Fig. 2). Compared with normal-weight participants (BMI $<24 \mathrm{~kg} / \mathrm{m}^{2}$ ) in the lowest quintile of GRS, obese participants (BMI $\geq 28 \mathrm{~kg} / \mathrm{m}^{2}$ ) in the highest quintile of GRS had an OR of 4.91 (95\% CI 2.2210.88), while obese status had an OR of 2.25 (95\% CI 1.64-3.08) compared with normal-weight status. Participants who had a positive family history of type 2 diabetes and were in the highest quintile of GRS had a dramatically higher disease risk (OR 20.52, 95\% CI 10.67-39.48) compared with those without a family history in the lowest quintile of GRS. Moreover, positive family history of diabetes alone increased the type 2 diabetes risk by 4.85 -fold ( $95 \%$ CI 3.69-6.38). However, no significant interaction between the GRS and conventional risk factors including BMI $(p=0.36)$, family history of diabetes $(p=0.30)$, smoking $(p=0.85)$, alcohol use $(p=0.72)$ and physical activity $(p=0.75)$ was observed for type 2 diabetes risk. Interestingly, we observed nominally significant interactive effects between the GRS and BMI on fasting glucose ( $p=0.04$ for interaction) and $\mathrm{HbA}_{1 \mathrm{c}}(p=0.03$ for interaction) levels (Fig. 1). Fasting glucose and $\mathrm{HbA}_{1 \mathrm{c}}$ significantly increased across quintiles of the GRS among obese ( $n=416 ; p=0.04$ and $p=0.02$, respectively) or overweight participants (BMI 24 to $<28 \mathrm{~kg} / \mathrm{m}^{2} ; n=1,088 ; p=0.001$ and $p=0.01$ for glucose and $\mathrm{HbA}_{1 \mathrm{c}}$, respectively), but not in normal-weight participants $(n=1,389 ; p \geq 0.40)$. There was no significant interactive effect between GRS and BMI on insulin $(p=0.86)$, HOMA-B $(p=0.56)$ or HOMA-S $(p=0.50)$.

As shown in Fig. 2, the GRS alone had a lower discriminatory ability for risk of type 2 diabetes (AUC $=$ 0.62). The AUC for the conventional clinical risk factors was 0.77 and was significantly increased to $0.79(p<0.001)$ after adding the GRS.

\section{Discussion}

In this population-based cohort of Han Chinese, we found that a GRS, based on 17 genetic variants from recent GWAS, was significantly associated with type 2 diabetes and hyperglycaemia, as well as with diabetes-related
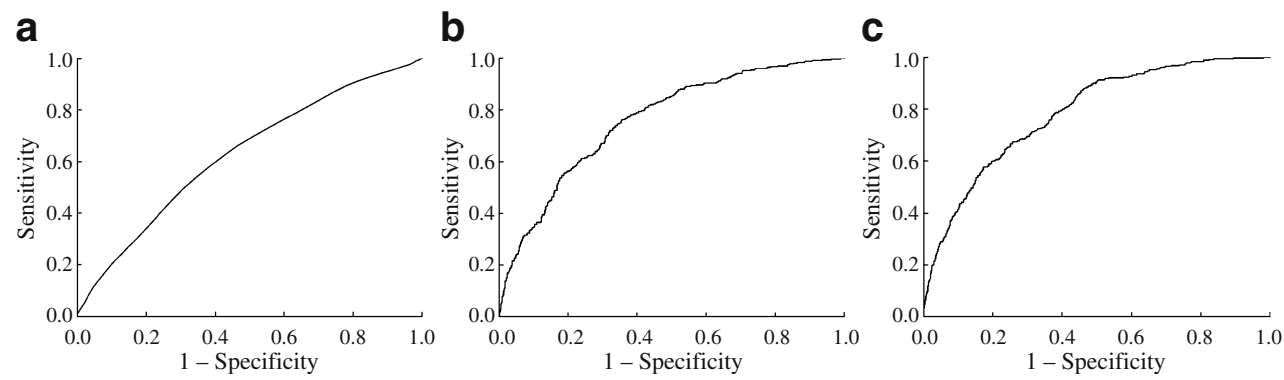

Fig. 2 Receiver operating characteristic curves for type 2 diabetes. Graphs show curves based on (a) GRS (AUC $=0.62)$, (b) conventional risk factors including age, sex, region, BMI, family history of diabetes, smoking, alcohol use, physical activity, HDL-cholesterol and log-transformed triacylglycerol $(\mathrm{AUC}=0.77)$ and $(\mathbf{c}) \mathrm{GRS}$ and conventional risk factors combined $(\mathrm{AUC}=0.79)$ 
traits, especially beta cell function. Combining the GRS with conventional risk factors slightly but significantly improved case-control discrimination for type 2 diabetes risk.

Several previous studies have shown that GRS could be used as a simple proxy of an individual's genetic predisposition to type 2 diabetes [2-6]. Consistent with this, we found that each additional risk allele increased the risk of type 2 diabetes by approximately $18 \%$ and that participants with a GRS $\geq 19.0(8.87 \%)$ had a 4.58 -fold increased risk of type 2 diabetes compared with those with a GRS $\leq 11.0(7.63 \%)$. Notably, the proportion of high-risk individuals with the highest GRS was much greater in this study than that in a previous study in white Europeans [2], which might be explained by ethnic differences in genetic predisposition to type 2 diabetes.

For the diabetes-associated quantitative traits, the strongest association was observed between the GRS and HOMA-B, supporting the notion that most of the genetic risk variants identified so far exert their diabetogenic effects through impaired beta cell function [1]. Fasting glucose and $\mathrm{HbA}_{1 \mathrm{c}}$ levels increased across quintiles of GRS among obese or overweight participants, but not in normal-weight participants, suggesting that the associations between GRS and these diabetes-related traits might be subject to modification by BMI. A previous study also observed a stronger effect of GRS on type 2 diabetes risk in obese individuals than in normal-weight individuals [5]. However, our results need to be confirmed in future studies because the interactions with fasting glucose and $\mathrm{HbA}_{1 \mathrm{c}}$ were only nominally significant, and no interaction between GRS and BMI was observed for type 2 diabetes risk.

As observed in the previous studies [2-6], genetic information slightly but significantly improved case-control discrimination of type 2 diabetes risk. A possible reason for the minimal improvement of the discriminatory value is that genetic variants might exert their diabetogenic effects through conventional risk factors such as BMI. Also, family history of diabetes as a risk factor already contains some genetic information provided by the GRS. The discriminatory ability of genetic variants may be improved when more susceptibility loci are included in future.

Several limitations of this study warrant mention. First, the cross-sectional nature limits the study to discriminatory analysis. Second, this study had insufficient power to detect diabetes association of low-risk variants (ESM Table 2). Well-powered studies in Chinese populations are required for a definite conclusion. Third, no established $\beta$ coefficient values for these genetic variants could be used to calculate a weighted GRS in this study. However, evidence suggests that there was no obvious difference in the results from the simple count and weighted GRS [5].
In conclusion, the GRS based on the 17 diabetesassociated variants modestly but significantly improved discrimination of conventional risk factors for type 2 diabetes in this Han Chinese population.

Acknowledgements This study was financially supported by the Chief Scientist Program of Shanghai Institutes for Biological Sciences, Chinese Academy of Sciences (SIBS2008006), the National Natural Science Foundation of China (30930081), the Ministry of Science and Technology of China (973 Program, Grant number 2006CB503900; 863 Program, Grant number 2007AA02Z332; and International Collaboration Program, Grant number 2008DFA31960) and the Chinese Academy of Sciences (The Knowledge Innovation Program, Grant number KSCX1-YW-02). We are grateful to all participants of the Study on Nutrition and Health of Aging Population in China. We also thank X. Pang, Z. Zhang, S. Jiao, H. Liu and S. Zhou from the Beijing and Shanghai Centers for Disease Control and Prevention for participating in the field work, and our colleagues W. Gan and G. Zong from the Institute for Nutritional Sciences for their part in the laboratory analyses.

Duality of interest The authors declare that there is no duality of interest associated with this manuscript.

\section{References}

1. McCarthy MI, Zeggini E (2009) Genome-wide association studies in type 2 diabetes. Curr Diab Rep 9:164-171

2. Lango H, the UK Type 2 Diabetes Genetics Consortium, Palmer CNA et al (2008) Assessing the combined impact of 18 common genetic variants of modest effect sizes on type 2 diabetes risk. Diabetes 57:3129-3135

3. Lyssenko V, Jonsson A, Almgren P et al (2008) Clinical risk factors, DNA variants, and the development of type 2 diabetes. N Engl J Med 359:2220-2232

4. van Hoek M, Dehghan A, Witteman JCM et al (2008) Predicting type 2 diabetes based on polymorphisms from genome-wide association studies: a population-based study. Diabetes 57:31223128

5. Cornelis MC, Qi L, Zhang C et al (2009) Joint effects of common genetic variants on the risk for type 2 diabetes in U.S. men and women of European ancestry. Ann Intern Med 150:541-550

6. Sparsø T, Grarup N, Andreasen C et al (2009) Combined analysis of 19 common validated type 2 diabetes susceptibility gene variants shows moderate discriminative value and no evidence of gene-gene interaction. Diabetologia 52:1308-1314

7. Ye X, Yu Z, Li H, Franco OH, Liu Y, Lin X (2007) Distributions of $\mathrm{C}$-reactive protein and its association with metabolic syndrome in middle-aged and older Chinese people. J Am Coll Cardiol 49:1798-1805

8. Wu Y, Li H, Loos RJ et al (2008) Common variants in CDKAL1, $C D K N 2 A / B, I G F 2 B P 2$, SLC30A8, and HHEX/IDE genes are associated with type 2 diabetes and impaired fasting glucose in a Chinese Han population. Diabetes 57:2834-2842

9. Qi Q, Li H, Loos RJ et al (2009) Common variants in KCNQ1 are associated with type 2 diabetes and impaired fasting glucose in a Chinese Han population. Hum Mol Genet 18:3508-3515

10. Qi Q, Wu Y, Li H et al (2009) Association of GCKR rs780094, alone or in combination with GCK rs1799884, with type 2 diabetes and related traits in a Han Chinese population. Diabetologia 52:834-843 